\title{
Phenolic residues in spruce galactoglucomannans improve stabilization of oil-in-water emulsions
}

\section{Lehtonen, Mari}

2018-02-15

Lehtonen , M , Merinen , M , Kilpeläinen , P O , Xu , C , Willför , S M \& Mikkonen , K S 2018

, 'Phenolic residues in spruce galactoglucomannans improve stabilization of oil-in-water

pÿemulsions ' , Journal of Colloid and Interface Science , vol. 512 , pp. 536547 . https://doi.org/10.1016/j.jcis.2017.1

http://hdl.handle.net/10138/308980

https://doi.org/10.1016/j.jcis.2017.10.097

cc_by_nc_nd

acceptedVersion

Downloaded from Helda, University of Helsinki institutional repository.

This is an electronic reprint of the original article.

This reprint may differ from the original in pagination and typographic detail.

Please cite the original version. 


\section{Phenolic residues in spruce galactoglucomannans}

\section{2 improve stabilization of oil-in-water emulsions}

3 M. Lehtonen, ${ }^{\mathrm{a}}$ M. Merinen, ${ }^{\mathrm{a}}$ P. O. Kilpeläinen, ${ }^{\mathrm{b}}$ C. Xu, ${ }^{\mathrm{c}}$ S. M. Willför ${ }^{\mathrm{c}}$ and K. S. Mikkonen ${ }^{\mathrm{a} *}$

4 a. Department of Food and Environmental Sciences, University of Helsinki, FI-00014, Helsinki,

5 Finland

$6 \quad$ b. Natural Resources Institute Finland (Luke), FI-00790, Helsinki, Finland

7 c. Johan Gadolin Process Chemistry Centre, c/o Laboratory of Wood and Paper Chemistry, Åbo Akademi 8 University, FI-20500, Turku, Finland.

9 "Corresponding Author, Email. kirsi.s.mikkonen@helsinki.fi, Tel. +358-2941-40526

11 ABSTRACT

12 Hypothesis. Amphiphilic character of surfactants drives them at the interface of dispersed systems, 13 such as emulsions. Hemicellulose-rich wood extracts contain assemblies (lignin-carbohydrate 14 complexes, LCC) with natural amphiphilicity, which is expected to depend on their chemical 15 composition resulting from the isolation method. Lignin-derived phenolic residues associated with 16 hemicelluloses are hypothesized to contribute to emulsions' interfacial properties and stability.

17 Experiments. We investigated the role of phenolic residues in spruce hemicellulose extracts in the 18 stabilization of oil-in-water emulsions by physical and chemical approach. Distribution and changes 
19 occurring in the phenolic residues at the droplet interface and in the continuous phase were studied during

20 an accelerated storage test. Meanwhile, the physical stability and lipid oxidation in emulsions were

21 monitored.

22 Findings. Naturally associated lignin residues in GGM act as vehicles for anchoring these

23 hemicelluloses into the oil droplet interface and further enable superior stabilization of emulsions. By

24 adjusting the isolation method of GGM regarding their phenolic profile, their functionalities, especially

25 interfacial behavior, can be altered. Retaining the native interactions of GGM and phenolic residues is

26 suggested for efficient physical stabilization and extended protection against lipid oxidation. The results

27 can be widely applied as guidelines in tailoring natural or synthetic amphiphilic compounds for 28 interfacial stabilization.

29 Keywords

30 Spruce galactoglucomannans, phenolic residues, emulsion stability, lipid oxidation

\section{Abbreviations}

32 eTMP ethanol precipitated galactoglucomannan (GGM) from thermomechanical pulping, LCC lignin-

33 carbohydrate complexes, OHB hydroxybenzoates, OHC hydroxycinnamates, PE spruce phenol extract,

34 PHWE GGM from pressurized hot-water extraction, PR phenolic residue, PV peroxide value, sTMP

35 spray-dried GGM from thermomechanical pulping.

\section{Introduction}


37 To deliver and protect lipophilic compounds in both technical applications and foods, lipids are 38 preferably dispersed into polar media, most often water. This formulation - emulsion - enhances the 39 physical properties of the product and enables efficient delivery of bioactive compounds, for example.

40 To mix two immiscible liquids and stabilize emulsions, additional components are needed, namely, 41 emulsifiers and stabilizers. ${ }^{1}$ Small-molecular amphiphilic compounds, such as phospholipids and 42 organosulphates, adsorb readily at the emulsion interface and decrease the energy needed for 43 emulsification. However, the interface is sensitive to environmental changes, such as the presence of 44 other amphiphilic compounds or mechanical forces. In addition to classical surfactants, macromolecules, 45 such as proteins and polysaccharides, are used as emulsifiers and/or stabilizers. The advantage of using amphiphilicity, or via increment of steric repulsion and/or continuous phase viscosity. ${ }^{4-5}$ Examples include proteinous gum Arabic and corn fiber gum, and very large polysaccharides, such as pectin and xanthan. ${ }^{6-9}$ Pickering-type stabilization has also been proposed for polysaccharides. ${ }^{10-11}$ That is, nano54 sized particles adsorb to the interface, creating a rigid barrier against coalescence and transfer of compounds between the dispersed and continuous phases. such as transition metals. Lipid oxidation decreases the functionality and nutritional value of lipids and produces off-flavors. Moreover, oxidation causes physical changes to the emulsion structures, e.g., 59 polymerization of lipids and eruption of emulsions. ${ }^{12}$ Thus, lipids should be protected against oxidation, and at the same time, the interfacial structure may be protected. The rate of oxidation is dependent on 
61 the amount and type of oil, emulsifiers, and stabilizers, and on the partitioning of the emulsifier and

62 stabilizer between the interface and continuous phase..$^{2,13-14}$

63 Approaches to stabilizing interfaces include thickening the interface or altering its charge with 64 multilayer coatings ${ }^{14-16}$ or incorporating antioxidants, such as polyphenols, to the interface. ${ }^{17}$ These 65 multi-step techniques principally use electrostatic deposition of polymer layers. That is, oppositely 66 charged polymers are layered one by one on the droplet surface. This technique is laborious and requires 67 great control of the process parameters, such as $\mathrm{pH}$ and ion strength, thus leading to costly production.

68 Biorefineries are currently being developed for profitable isolation of various lignocellulosic fractions 69 into precursors of bio-based chemicals and materials. Availability of potential new renewable raw materials has extended the possibilities to explore their suitability for industrial applications; however, in many cases, their applicability or sustainability may be limited due to the need for hazardous solvents or reagents in biomass derivatization. Conversion of non-food biomass into a source of energy or raw materials for technical products often involves a sequence of chemical derivatization reactions aided by 74 solvents and reagents. Within the biorefinery approach, biomass isolation techniques are being developed to control the composition and functions of isolated fractions. In this paper, we present a green chemistry approach towards dispersing agents by exploring and exploiting the natural characteristics of softwood extracts, namely, spruce $O$-acetyl-galactoglucomannans (GGM), wood-derived hemicelluloses that are still widely untapped, as multifunctional stabilizers.

We have previously shown that spruce GGM, abundant future forest biorefinery products that are relatively small, linear chain polysaccharides, adsorb at the lipid droplet interface and stabilize it against

81 physical breakdown and lipid oxidation. ${ }^{18-20}$ However, the action mechanisms are yet to be discovered. 82 GGM are envisioned as multifunctional, sustainable, natural stabilizers that could replace several less 83 effective additives and be applied in various types of industrial dispersions, including food, cosmetics 84 and pharmaceuticals after safety evaluations (Pitkänen et al., submitted) and in technical applications, 85 e.g. coatings and chemicals. 
GGM are hemicelluloses that consist of chains of $(1 \rightarrow 4)$-linked $\beta$-D-mannopyranosyl and $\beta$-D-

87 glucopyranosyl units with single $(1 \rightarrow 6)$-linked $\alpha$-D-galactopyranosyl units attached to mannose..$^{22}$ Part of the secondary hydroxyl groups of mannose units are acetylated, which introduces hydrophobic sites to the structure. Yet, the backbone of GGM lacks clear non-polar parts that would give GGM an amphiphilic nature. In softwood, hemicelluloses may be associated or partially covalently bound to lignin, forming lignin-carbohydrate complexes (LCC). ${ }^{23}$ Lignin-originating phenolic residues (PR) are likely to accompany hemicelluloses during the isolation process and provide additional characteristics to GGM. repulsion and/or Pickering-type stabilization by GGM assemblies. We have previously shown that GGM adsorbs at the oil droplet interface, but the driving force enabling adsorption is not known. ${ }^{18}$ At high GGM concentrations, stabilization also occurs via increment of viscosity. ${ }^{19}$ GGM also inhibits lipid oxidation both in oil-in-water emulsions and in microcapsules. ${ }^{20,24}$

The isolation method of hemicelluloses affects both the content and composition of hemicelluloses and accompanying PR. For example, by altering the temperature and extraction time in flow-through pressurized hot water extraction, the composition and size of recovered hemicelluloses and lignin can be controlled. ${ }^{25-27}$ However, the role of PR for the functionality of GGM is largely unknown; their presence may be either beneficial or detrimental, depending on the application.

The wide availability of raw materials, the economical isolation processes, and the multifunctionality of GGM make them a highly attractive stabilizer alternative for industrial use. To understand the features of GGM and enable development of applications, knowledge of their function mechanisms is needed. The aim of this study was to demonstrate the role of naturally occurring lignin-originating PR in GGM in the stabilization of oil-in-water emulsions. Thereby we illustrate the interaction and interfacial

109 functionality of lipophilic structures bound with hydrophilic polysaccharide tails. GGM isolated with 110 different techniques and having either hemicellulose-phenolic compound complexes or added phenolic 
111 extract were compared for their stabilization capacity. Partitioning of PR between the interface and

112 continuous phase of emulsions and the changes occurring in them were determined during an accelerated

113 storage test. Meanwhile, physical stability and lipid oxidation in emulsions was monitored. This study

114 reveals that GGM-phenolic complexes play a key role in emulsion stabilization.

\section{Materials and methods}

$116 \underline{\text { Materials }}$

117 GGM, a natural mixture containing variety of galactoglucomannans and wood extractives, were

118 obtained in powder form from process water of a Finnish pulp mill in an industrial-scale isolation trial

119 after spray-drying (sTMP) or after ethanol precipitation (eTMP) ${ }^{28-29}$ and as aqueous concentrate from

120 pressurized hot water flow-through extraction $(\mathrm{PHWE})^{25}$. The average molar mass of sTMP and eTMP 121 were $20000-30000 \mathrm{~g} / \mathrm{mol}^{18}$ and that of PHWE GGM below $10000 \mathrm{~g} / \mathrm{mol}^{19}$. Lignan-rich spruce phenol

122 extract (PE) was isolated from spruce knotwood according to Willför et al. ${ }^{30}$ Rapeseed oil, consisting of $12360 \%$ of monounsaturated, $31 \%$ of polyunsaturated, and $4 \%$ of saturated fatty acids (Bunge Finland Oy, 124 Raisio, Finland), was purchased from a supermarket and purified from natural anti- and pro-oxidants by 125 adsorption chromatography according to a previously described method. ${ }^{20,31}$

\section{Emulsion preparation}

127 Emulsions containing 5 wt.-\% of stripped rapeseed oil and 1 wt.-\% of GGM were prepared by high-

128 pressure homogenization according to a previously described method. ${ }^{20}$ For the addition of spruce 129 phenol extract (PE) to eTMP emulsion, PE was dissolved into acetone $(30 \mathrm{mg} / \mathrm{mL})$ and then dispersed 130 into 1 wt.-\% GGM solution before emulsification to yield $5.5 \mathrm{mg}$ added phenolic compounds in $100 \mathrm{~g}$ 131 emulsion. The total phenol content of spruce phenol extract was estimated using the Folin-Ciocalteu 
132 method ${ }^{32}$ and the amount of added extract was adjusted in order to double the amount of phenolic

133 compounds in the emulsions compared to samples with plain eTMP GGM.

\section{$134 \quad$ Accelerated storage test}

135 For the accelerated storage test, $100 \mathrm{~g}$ of emulsion was stored in a $250-\mathrm{mL}$ glass bottle at $40{ }^{\circ} \mathrm{C}$ in the 136 dark up to 2.5 months. Stability of the emulsion was monitored several times during the first two weeks

137 of storage and then in intervals of two weeks for a total of 11 weeks. At each sampling, a few drops of

138 emulsion were withdrawn for determining droplet size distribution and for visual investigation with 139 optical microscopy. At the same sampling, $0.5 \mathrm{~g}$ of emulsion was withdrawn for the analysis of peroxide 140 value and polymerized lipids. For monitoring the changes occurring in PR, $30 \mathrm{~g}$ of emulsion was 141 withdrawn after 0,7 and 14 days of storage. Before each sampling, the emulsion was mixed by turning 142 the containers upside down ten times. For the analysis of volatile oxidation products, $1.5 \mathrm{~g}$ of fresh 143 emulsion was placed in $20-\mathrm{mL}$ glass vials $(75.5 \times 22.5 \mathrm{~mm})$, sealed with caps, and the vials were stored 144 at $40^{\circ} \mathrm{C}$ in the dark. At each sampling, three replicate samples were withdrawn $(\mathrm{n}=3)$.

\section{Droplet size distribution}

146 The droplet size distribution was characterized by static light scattering using a Mastersizer Hydro

1473000 SM (Malvern Instruments Ltd, Worcestershire, UK). Before each sampling, the emulsion was 148 mixed gently by turning the container upside down ten times. Interfacial area was calculated from the 149 average droplet size and volume fraction of the added oil.

$150 \quad$ Microscopy

151 The emulsion morphology was characterized using optical microscopy (AxioScope A1, Carl Zeiss

152 Inc., Oberkochen, Germany). Before each sampling, the emulsion was mixed gently by turning the 153 container upside down ten times. 
154 Partitioning of emulsions

155 Emulsions were partitioned into aqueous and creamed phases by centrifugation. ${ }^{18} 30 \mathrm{~g}$ of emulsion 156 was centrifuged at $24000 \mathrm{~g}$ at RT for $15 \mathrm{~min}$. The creamed phase was collected and the residue was

157 centrifuged for additional $5 \mathrm{~min}$. The second creamed phase was combined with the first one for the 158 analysis of adsorbed GGM fraction. $1 \mathrm{~mL}$ of continuous phase was collected for the analysis of the non159 adsorbed GGM fraction. Both the creamed phase and continuous phase were subjected to further analysis 160 (Fig. 1).

161 Determination of phenolic residues by UHPLC-DAD-FLD

162 Distribution and changes occurring in interfacial and dispersed free and bound phenolic residues of

163 GGM were determined in order to evaluate their possible contribution to emulsion stability.

164 The creamed phase was divided into two equal portions. The adsorbed GGM were precipitated with

$16580 \mathrm{~mL}$ of $80 \%$ ethanol, which is also compatible for the extraction of free phenolic compounds (Fig. 1).

166 Released lipids were removed from the aqueous phase by extraction with $80 \mathrm{~mL}$ of iso-octane. The

167 organic phase was removed and the remaining aqueous phase was centrifuged (10 min at $24000 \mathrm{~g}$ ).

168 Supernatant and precipitated GGM were collected separately. The supernatant was evaporated to 169 dryness.

170 Non-adsorbed GGM in the continuous phase $(1 \mathrm{~mL})$ were precipitated with $4 \mathrm{~mL}$ of $80 \%$ ethanol.

171 Remaining lipids were removed by extraction with $5 \mathrm{~mL}$ of iso-octane. The aqueous phase and 172 precipitate were separated by centrifugation (10 min at $3000 \mathrm{~g}$ ). Supernatant and precipitated GGM were 173 collected separately. The supernatant was evaporated to dryness.

174 The hydrolyses and analysis of PR were performed according to a previously described method using 175 the same equipment and reagents. ${ }^{20}$ The analysis included fractions of ethanol-soluble free PR, ester176 bound PR to ethanol-soluble GGM, ester-bound PR to ethanol-precipitated GGM and glycosidically 
177 bound or otherwise entrapped PR in GGM (Fig. 1). All the analyses were performed in triplicates ( $\mathrm{n}=$

178 3). The results were expressed as $\mu \mathrm{g} / \mathrm{kg}$ of emulsion. The average and standard deviation were calculated

179 across three analytical replicates $(\mathrm{n}=3)$.

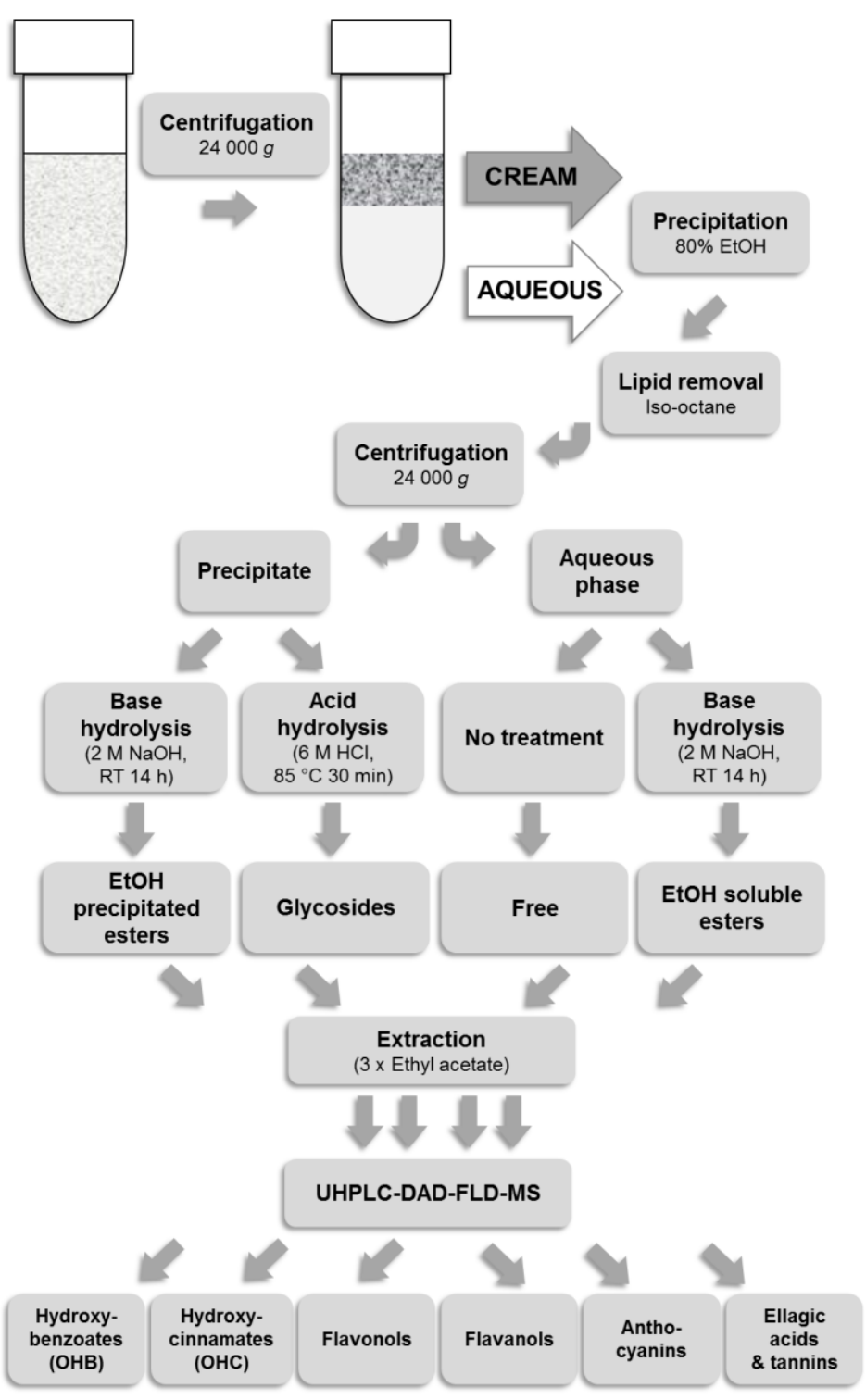

181 Figure 1. Sample preparation for the analysis of adsorbed and non-adsorbed phenolic residues. Each

182 creamed phase and aqueous phase provided results of six different compound groups in four different

183 fractions. The analysis was performed in triplicates.

184 Determination of peroxide value 
185 The primary oxidation of the emulsions was evaluated by the total content of hydroperoxides in the

186 lipid phase determined by the peroxide value (PV). The analysis was performed according to a previously

187 described method ${ }^{20}$ where lipids were first released and extracted and then PV was determined according

188 to a ferric thiocyanate method. The average and standard deviation were calculated across three 189 analytical replicates $(\mathrm{n}=3)$.

190 Determination of hexanal by SHS-GC-FID

191 The secondary stage of lipid oxidation in emulsions was followed in terms of hexanal formation 192 according to a previously described static headspace gas chromatography (SHS-GC-FID) method. ${ }^{33}$ At 193 each sampling time, three replicate vials were withdrawn for the analysis. Hexanal contents were 194 reported as peak area per gram of emulsion (peak area/g emulsion). The average and standard deviation 195 were calculated across three analytical replicates $(n=3)$.

\section{Determination of polymerized lipids by SEC-RI}

197 Lipid oxidation in emulsions was also followed in terms of polymerization. Formed oligomeric lipids 198 and remaining monomers were determined utilizing size-exclusion chromatography (SEC) in

199 combination with refractive index (RI) detection, as described previously. ${ }^{20}$ The results were reported as 200 relative peak area proportions (\%). The average and standard deviation were calculated across three 201 analytical replicates $(\mathrm{n}=3)$.

\section{Results}

203 Droplet size distribution and morphology 
204 Physical stabilization of emulsions by studied GGM extracts were evaluated by droplet size 205 distributions. The structures of emulsions were also visualized by optical microscopy to reveal possible 206 breakdown.

207 Emulsions stabilized by PHWE GGM had a unimodal droplet size distribution with an average droplet 208 size D[3,2] of $0.4 \mu \mathrm{m}$ (Fig. 2). The droplet size distribution remained similar during six weeks of 209 accelerated storage test at $40{ }^{\circ} \mathrm{C}$. In sTMP GGM-stabilized emulsions, the $\mathrm{D}[3,2]$ was larger $(2.1 \mu \mathrm{m})$ 210 and increased to $3.4 \mu \mathrm{m}$ during storage. The eTMP GGM-stabilized emulsions showed bimodal droplet 211 size distribution and an increase in droplet size during storage. The addition of PE to eTMP GGM did 212 not significantly influence the droplet size distribution, as the eTMP+PE emulsions formed and aged in 213 a similar manner to those containing plain eTMP GGM, in terms of droplet size distribution. Optical 214 microscopy images confirmed the presence of mainly very small droplets of less than $1 \mu \mathrm{m}$ in diameter 215 in PHWE emulsions, whereas large droplets and flocculation were seen in sTMP emulsions. In eTMP 216 and eTMP+PE emulsions, droplets with a large size range were observed, which could be due to 217 coalescence or Ostwald ripening (Fig. 3).

218 The total oil-water interfacial area of PHWE emulsion was $800 \mathrm{~m}^{2} / \mathrm{kg}$ while that of sTMP emulsion 219 was $150 \mathrm{~m}^{2} / \mathrm{kg}$ (Fig. 4). No significant changes were observed in PHWE emulsion while the interfacial 220 area of sTMP emulsion decreased to $100 \mathrm{~m}^{2} / \mathrm{kg}$ during the first week of storage at $40{ }^{\circ} \mathrm{C}$. The interfacial 221 area of eTMP and eTMP+PE emulsions decreased continuously from $1300 \mathrm{~m}^{2} / \mathrm{kg}$ to $400 \mathrm{~m}^{2} / \mathrm{kg} \mathrm{during}$ 222 two weeks of storage. 

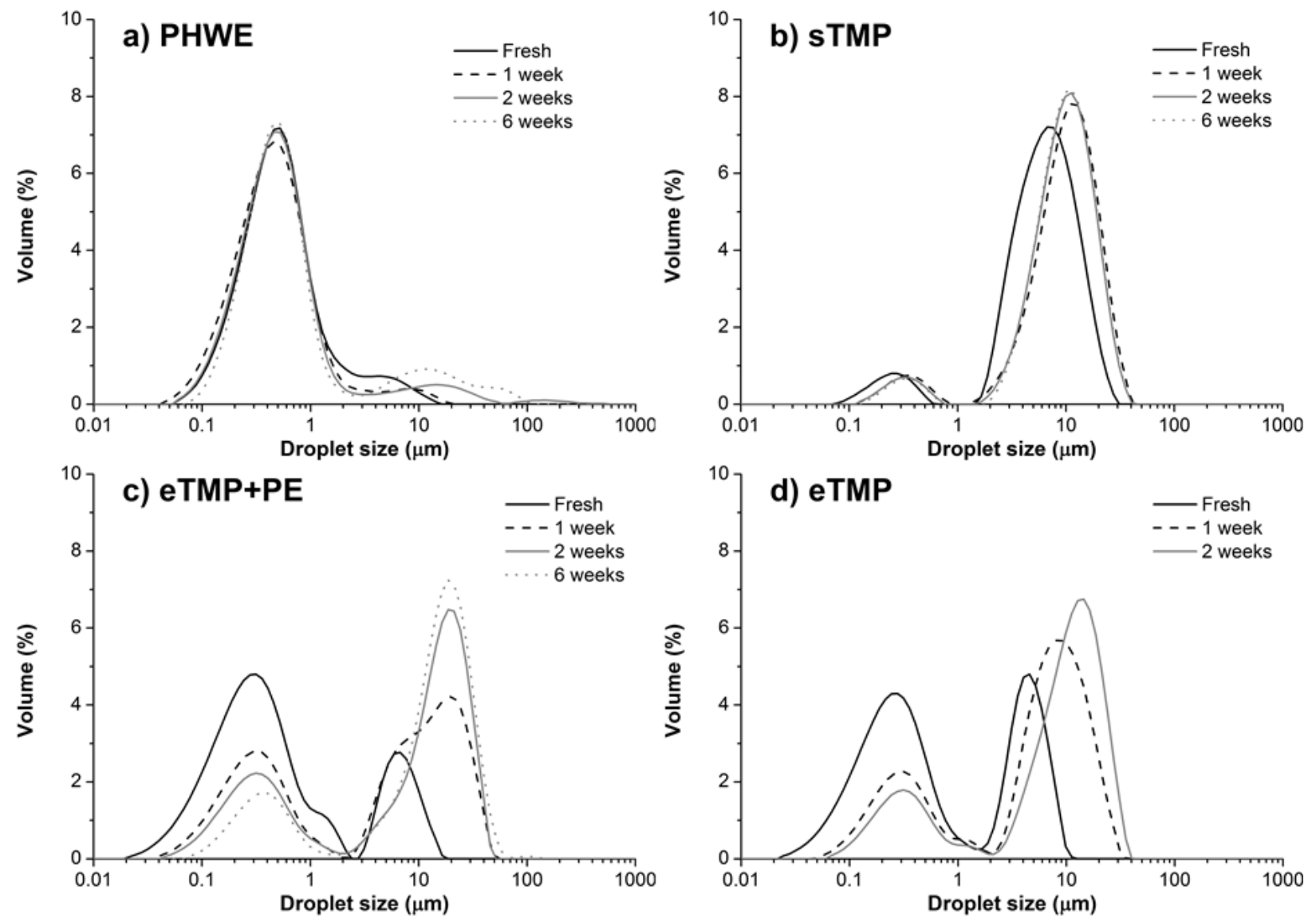

Figure 2. Droplet size distributions of emulsions with 5 wt.-\% rapeseed oil stabilized with 1 wt.-\% GGM

225 obtained from (a) pressurized hot water extraction (PHWE), (b) thermomechanical pulping after spray-

226 drying (sTMP), (c) thermomechanical pulping after ethanol precipitation and addition of spruce phenol

227 extract (eTMP + PE), and (d) eTMP as fresh and after $1-6$ weeks storage at $40{ }^{\circ} \mathrm{C}$. The graphs are the 228 averages of three replicate measurements. 

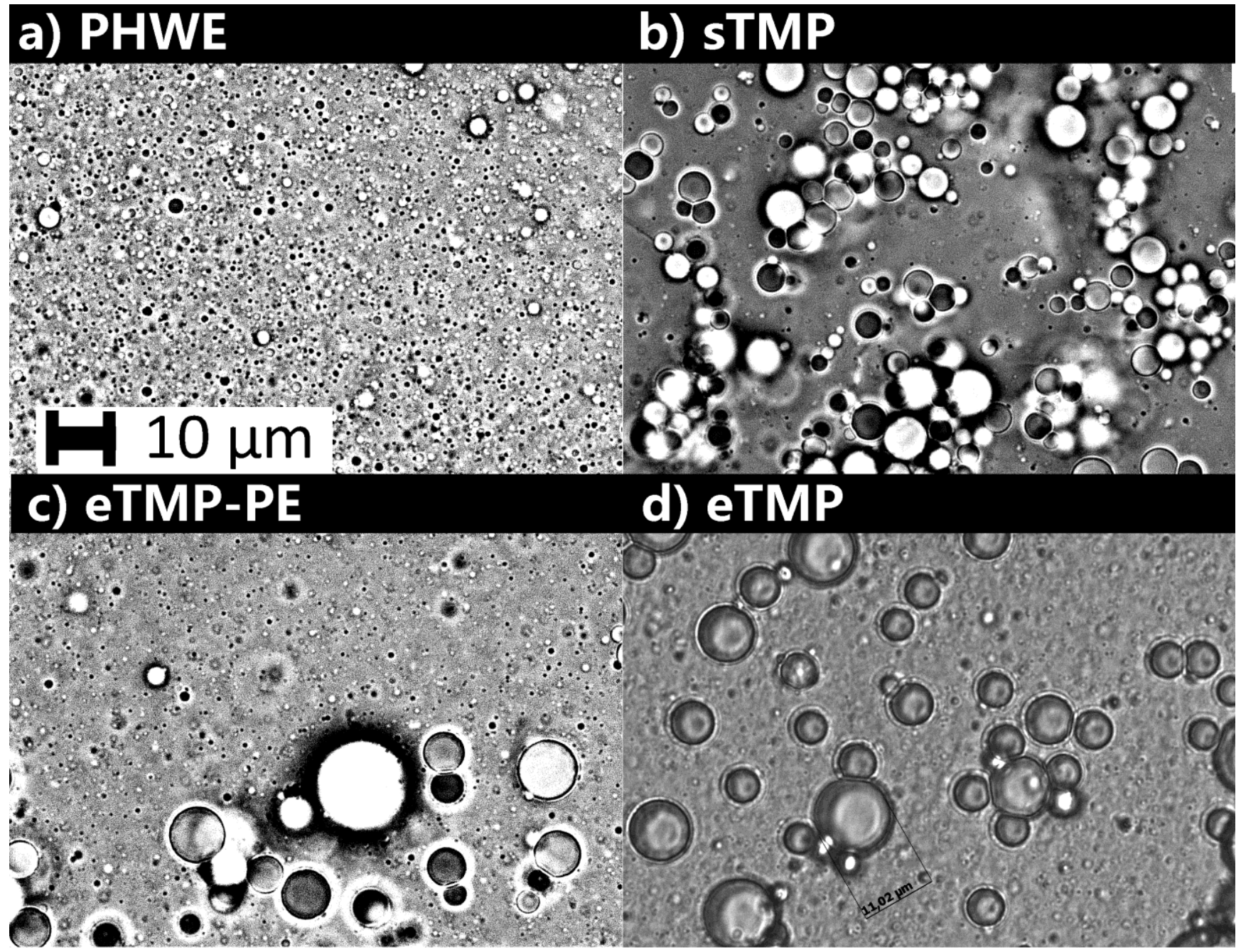

230 Figure 3. Optical microscopy images of emulsions with 5 wt.-\% rapeseed oil stabilized with 1 wt.-\%

231 GGM, stored at $40{ }^{\circ} \mathrm{C}$ for 2 weeks. GGM obtained from (a) pressurized hot water extraction (PHWE),

232 (b) thermomechanical pulping after spray-drying (sTMP), (c) thermomechanical pulping after ethanol

233 precipitation with addition of spruce phenol extract (eTMP + PE), and (d) eTMP without additives. 


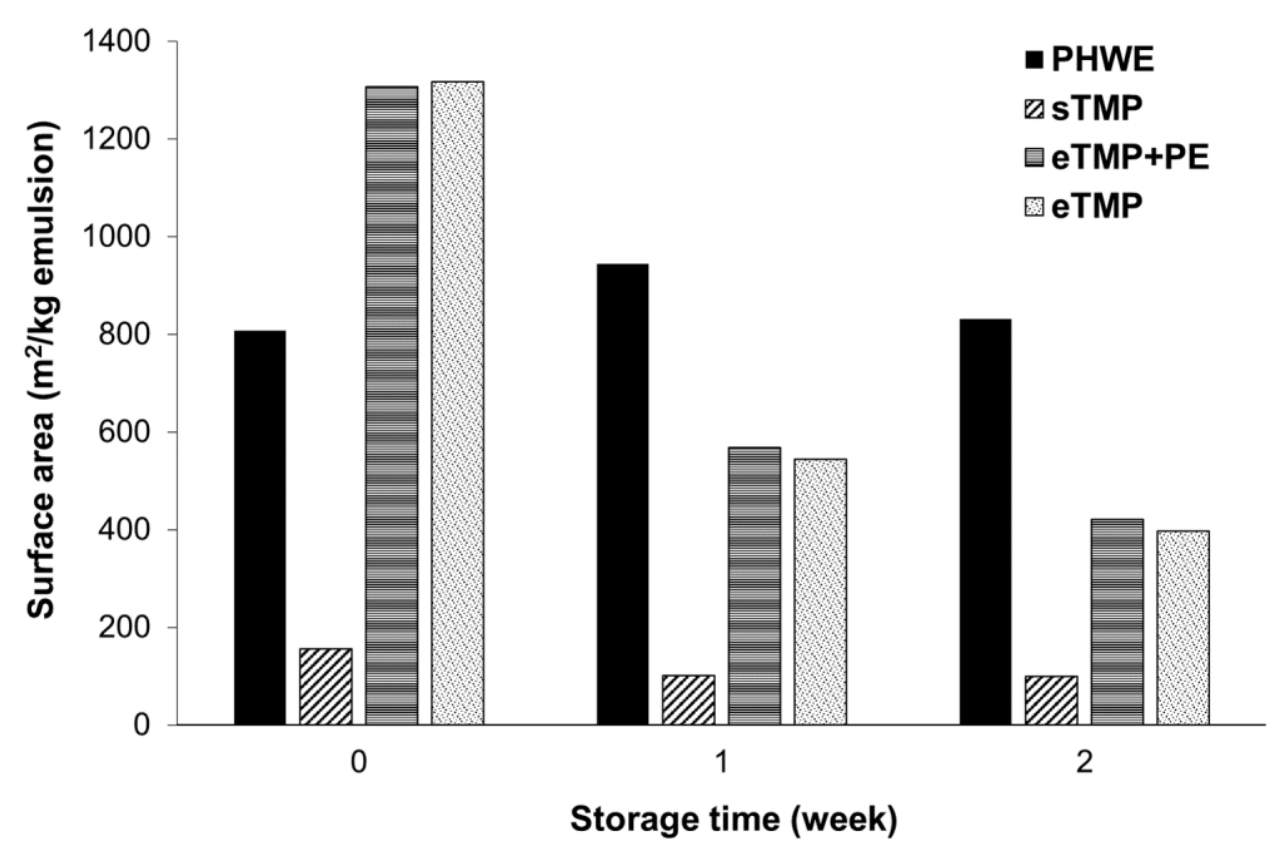

235 Figure 4. Total interfacial area of lipid droplets (5 wt.-\%) in GGM-stabilized (1 wt.-\%) emulsions during 236 accelerated storage test at $40{ }^{\circ} \mathrm{C}$. GGM obtained from pressurized hot water extraction (PHWE), 237 thermomechanical pulping after spray-drying (sTMP) and thermomechanical pulping after ethanol 238 precipitation (eTMP) and addition of spruce phenol extract (eTMP + PE).

Distribution of phenolic residues in emulsions

To understand how phenolic residues (PR) in GGM affect emulsion stability, their distribution between the interface and continuous phase was determined.

In all the studied emulsions, less than $0.1 \%$ of PR were located at the interface while the majority remained in the continuous phase (Fig. 5). The content of interfacial PR in PHWE GGM-stabilized emulsion was $2.6 \mu \mathrm{g} / \mathrm{kg}$ emulsion, while the content in the continuous phase was $111 \mathrm{mg} / \mathrm{kg}$ emulsion. The majority of the adsorbed PR (77\%) were ester bound to ethanol-soluble GGM fraction, but some of the free or weakly associated PR (21\%) were located at the interface. The continuous phase of PHWE emulsion had similar distribution of ester bound and free PR as at the interface though the contents were greater. The chemical structures of the adsorbed PR and those of remaining in the continuous phase were different. While ester-bound hydroxycinnamyls (OHC) (57\%) and hydroxybenzoyls (OHB) (32\%) were the main PR units at the interface, ester-bound OHB (62\%) and flavanols (35\%) were dominant in the 
251 continuous phase. In the free PR, the proportion of flavanols $(39 \%)$ and OHC (29\%) were greater at the 252 interface than in the continuous phase (19\% and $10 \%$, respectively) while the proportion of OHB was 253 far greater (71\%) in the continuous phase than at the interface $(32 \%)$.

254 In sTMP GGM-stabilized emulsion, the content of interfacial PR was $0.4 \mu \mathrm{g} / \mathrm{kg}$ emulsion and the 255 content of continuous phase PR was $59 \mathrm{mg} / \mathrm{kg}$ emulsion (Fig. 5). PR ester bound to ethanol-soluble 256 GGM and free PR comprised equal fractions (47\%) at the interface. Free PR were the most abundant 257 fraction (53\%) in the continuous phase. Flavanols constituted the majority (65-70\%) of the adsorbed 258 and continuous phase PR followed by OHB (24-26\%).

259 In eTMP+PE GGM-stabilized emulsion, PR of $1.7 \mu \mathrm{g} / \mathrm{kg}$ emulsion were adsorbed at the interface 260 while $41 \mathrm{mg} / \mathrm{kg}$ emulsion remained in the continuous phase (Fig. 5). PR ester bound to ethanol-soluble 261 GGM was the main fraction (54\%) at the interface, but also a fraction of free (21\%) and glycosidically 262 bound (22\%) PR were adsorbed. Free PR (57\%) and glycosidically bound PR (39\%) constituted the main 263 fractions in the continuous phase. Flavanols constituted the majority (53-65\%) of the adsorbed and 264 continuous phase PR followed by OHB (35-47\%).

265 As the content of PR in eTMP GGM was considered relatively low, ${ }^{20}$ that is, much lower than in sTMP 266 GGM, the distribution of PR in eTMP emulsions were not studied in more detail. 

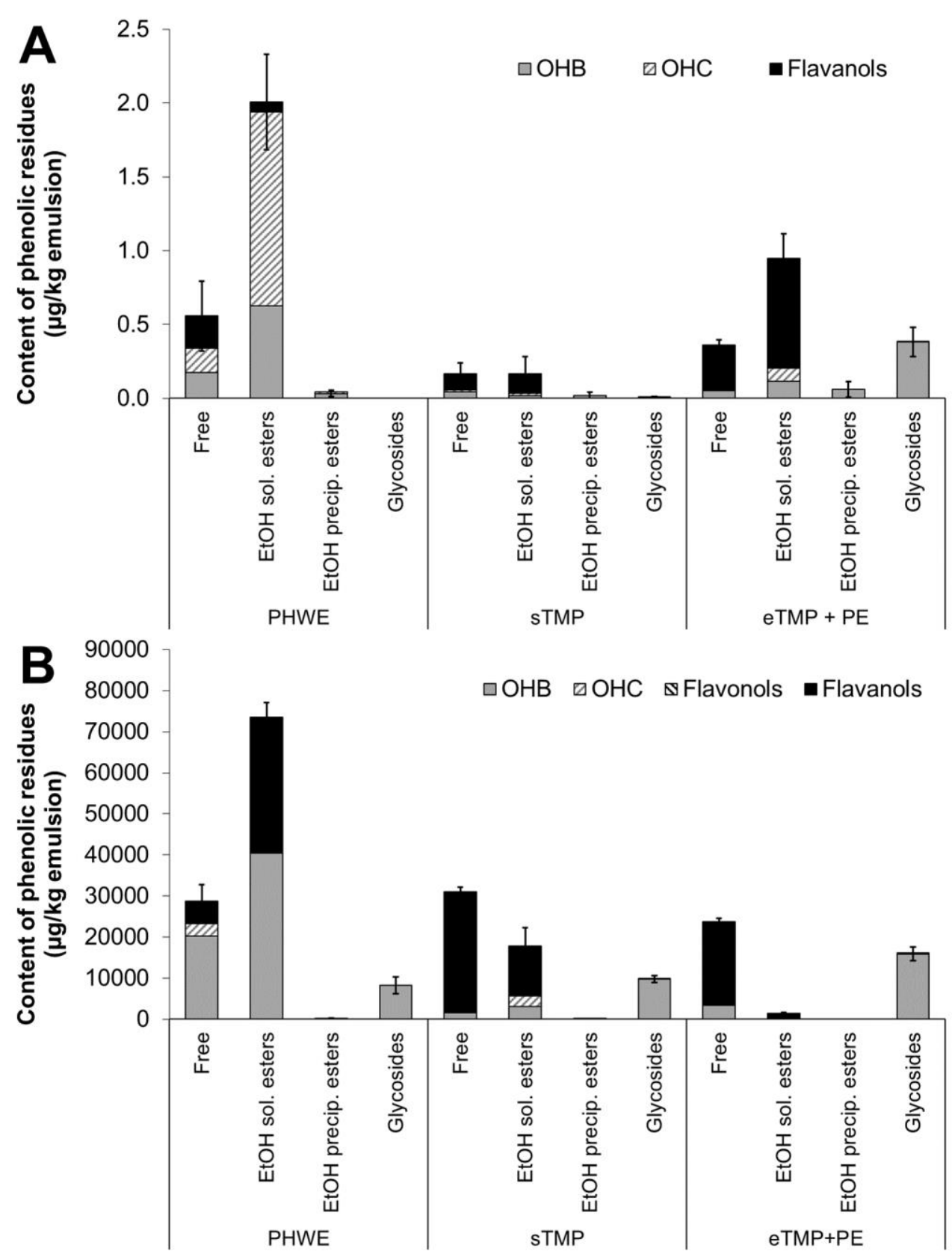

Figure 5. Composition of adsorbed (A) and non-adsorbed (B) phenolic residues in stripped rapeseed oil

269 (5 wt.-\%) emulsions stabilized by GGM (1 wt.\%). GGM obtained from pressurized hot water extraction 270 (PHWE), thermomechanical pulping after spray-drying (sTMP) and thermomechanical pulping after

271 ethanol precipitation and addition of spruce phenol extract (eTMP + PE). The graphs presenting 272 hydroxybenzoates (OHB), hydroxycinnamates (OHC), flavonols and flavanols are the averages of three 273 replicate analyses. 
274 Changes occurring in the phenolic residues during storage

275 In order to understand the role of phenolic residues (PR) in the physical and oxidative stabilization of 276 emulsions, changes occurring in the distribution and contents of PR were monitored during the first two 277 weeks of accelerated storage test.

278 Changes in the contents of PR were observed both in the interfacial and continuous phases throughout 279 the accelerated storage test (Fig. 6). In PHWE emulsion, the changes were more substantial in the 280 continuous phase than at the interface: a decrease of $55-62 \%$ in the total PR content was observed in the 281 continuous phase while the decrease was only $24 \%$ at the interface. Further changes were not observed 282 after one week of storage. The changes in the continuous phase occurred mainly in PR ester bound to 283 ethanol-soluble GGM (72\%) and at the interface in free PR (55\%). In the continuous phase, the decrease 284 was more pronounced in OHB (89\%) compared to flavanols (51\%). At the interface, the reduction was 285 similar for both OHB and flavanols (60-68\%).

286 In sTMP emulsions, changes also occurred mainly in the continuous phase PR: a continuous decrease 287 of $35 \%$ in their content was observed during two weeks. The changes occurred primarily in PR ester 288 bound to ethanol-precipitated GGM (84\%) and in PR ester bound to ethanol-soluble GGM (60\%). The 289 contents of all of the PR, OHB, OHC and flavanols decreased (51-87\%).

290 Unlike in the other emulsions, in eTMP+PE emulsion, changes occurred only in the interfacial 291 phenolic residues: a continuous decrease of $74 \%$ occurred over two weeks. The changes could be 292 observed in all of the studied fractions, being most abundant in glycosidically bound PR (87\%). The 293 greatest decrease occurred in OHB and OHC (87-94\%).

294 As the content of PR in eTMP GGM was relatively low, ${ }^{20}$ the changes occurring in their contents 295 during emulsion storage were not studied in more detail. 

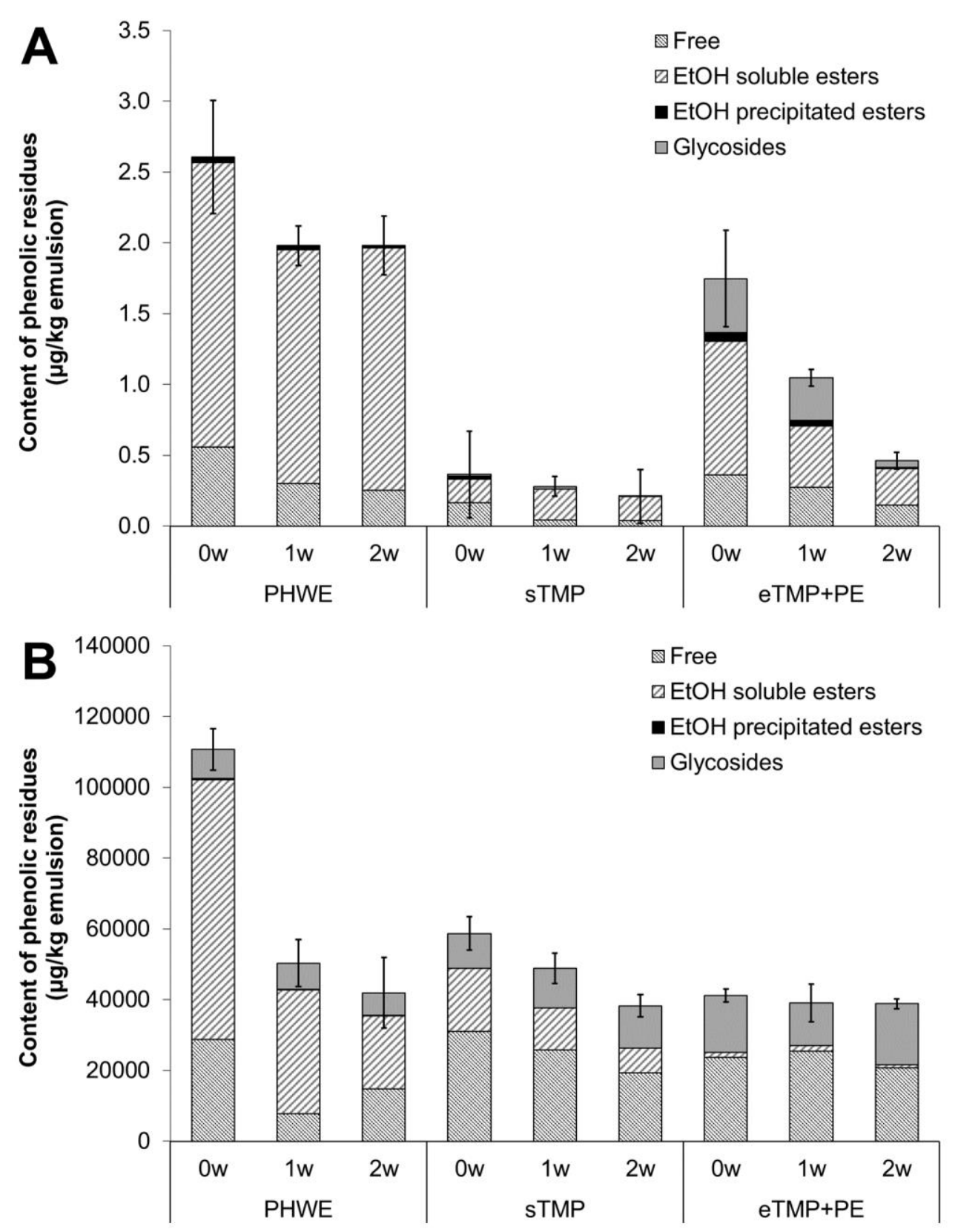

298 Figure 6. Changes occurring in the adsorbed (A) and non-adsorbed (B) phenolic residues in stripped 299 rapeseed oil (5 wt.-\%) emulsions stabilized by GGM (1 wt.\%) during accelerated storage test at $40{ }^{\circ} \mathrm{C}$.

300 GGM obtained from pressurized hot water extraction (PHWE), thermomechanical pulping after spray-

301 drying (sTMP) and thermomechanical pulping after ethanol precipitation and addition of spruce phenol

302 extract (eTMP + PE). The graphs are the averages of three replicate analyses. 

only after 9 weeks of storage at $40{ }^{\circ} \mathrm{C}$ (Fig. 7). In sTMP emulsion, hydroperoxides were detected after 2 weeks, and PV of $20 \mathrm{mEq} / \mathrm{kg}$ oil was measured after 6 weeks. eTMP GGM inhibited oxidation, but not as efficiently: hydroperoxides were detected after 4 days of storage and PV of $264 \mathrm{mEq} / \mathrm{kg}$ was measured

308 after two weeks. Addition of spruce phenol extract to eTMP GGM enhanced the stability of lipids:

309 hydroperoxides were detected after 9 days $(10 \mathrm{mEq} / \mathrm{kg}$ oil) and $\mathrm{PV}$ of $228 \mathrm{mEq} / \mathrm{kg}$ oil was measured 310 after 8 weeks of storage.

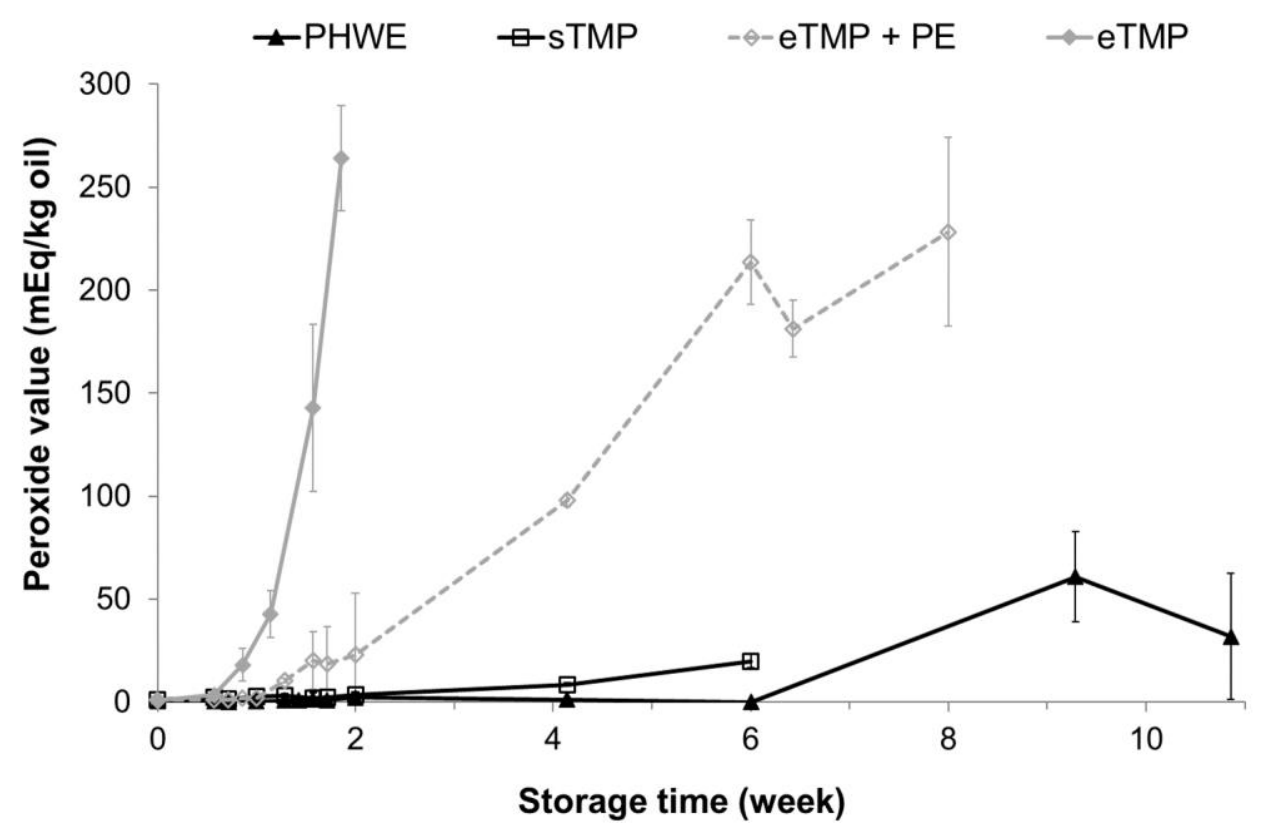

312 Figure 7. Formation of hydroperoxides in the stripped rapeseed oil phase (5 wt.\%) of GGM (1 wt.\%)

313 stabilized emulsions during accelerated storage test at $40{ }^{\circ} \mathrm{C}$. GGM obtained from pressurized hot water 314 extraction (PHWE), thermomechanical pulping after spray-drying (sTMP) and thermomechanical 315 pulping after ethanol precipitation (eTMP) and addition of spruce phenol extract (eTMP + PE). The 316 graphs are the averages of three replicate analyses. 
317 In PHWE and sTMP emulsions, hexanal was not detected during the first four weeks of storage (Fig.

318 8). As was seen in PV, spruce phenol extract added to eTMP GGM delayed the oxidation but not as 319 efficiently as PHWE GGM and sTMP GGM. In eTMP GGM-stabilized emulsion, hexanal was detected 320 after 2 weeks and was detected in eTMP+PE emulsions after 3 weeks. After 1.5 months of storage, the 321 samples in headspace vials were physically separated and thus volatile oxidation products were no longer 322 determined.

323 No polymerization or interaction products of the lipids were detected during the 2.5-month accelerated 324 storage test in any of the studied emulsions.

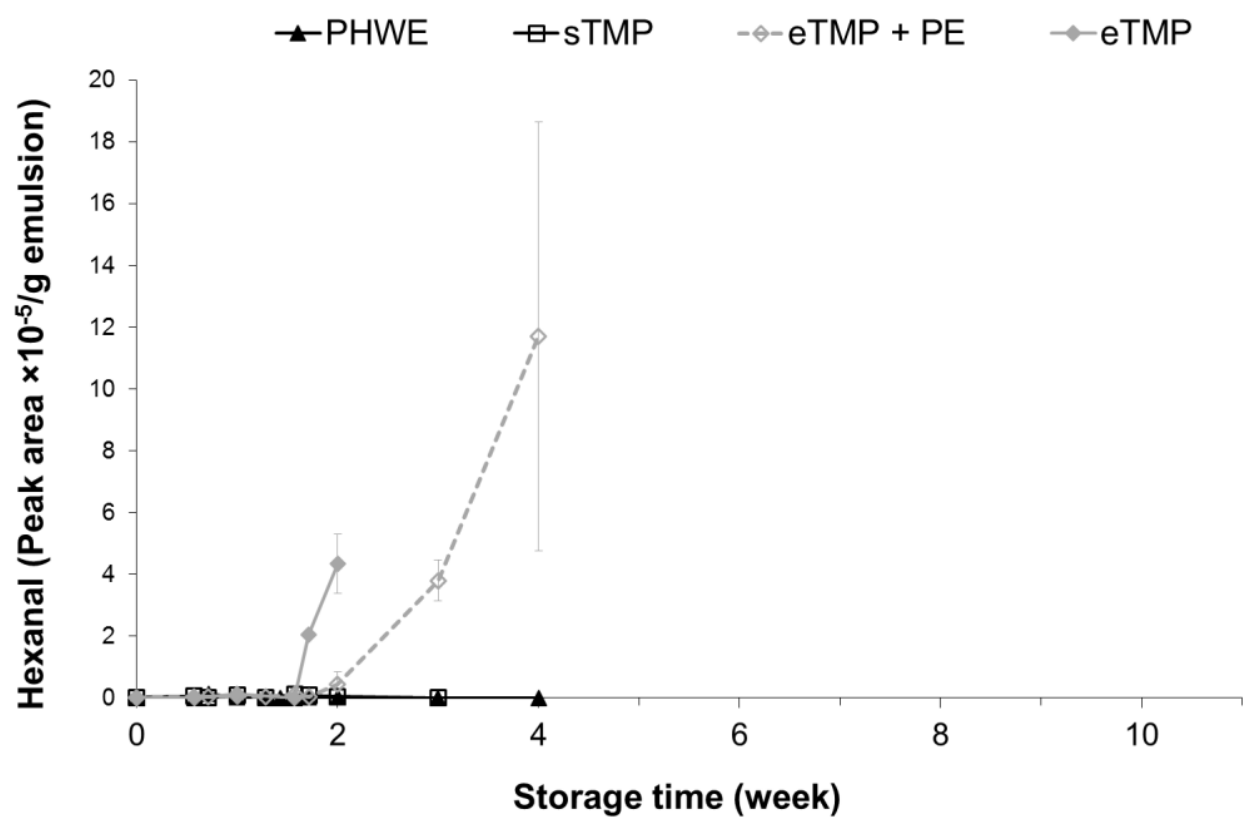

326 Figure 8. Formation of hexanal in the stripped rapeseed oil phase (5 wt.\%) of GGM (1 wt.\%) stabilized 327 emulsions during accelerated storage test at $40^{\circ} \mathrm{C}$. GGM obtained from pressurized hot water extraction 328 (PHWE), thermomechanical pulping after spray-drying (sTMP) and thermomechanical pulping after 329 ethanol precipitation (eTMP) and addition of spruce phenol extract (eTMP + PE). The graphs are the 330 averages of three replicate analyses. 
This study clearly demonstrates that GGM containing bound phenolic residues are more efficient emulsion stabilizers than purified (ethanol precipitated) GGM alone or with incorporated phenolic compounds. This applies both for the physical stability and for the protection of dispersed lipids against oxidation. The structure and composition of residual phenolic compounds (PR) in GGM define their ability to adsorb to the oil-water interface and stabilize it. In addition, PR are responsible for the remarkable oxidative stability of the dispersed lipids. These observations will be discussed further.

Although only small portion of PR adsorbed at the interface, they had a remarkable effect on emulsion morphology and stability. According to our earlier findings, the surface load of eTMP GGM in corresponding $5 \%$ oil-in-water emulsion was $0.8 \mathrm{mg} / \mathrm{m}^{2} .{ }^{18}$ This surface load corresponds to approximately $10 \%$ of GGM present in the emulsion. However, in the present study, less than $1 \%$ of the PR present in the emulsions were located at the interface. As the molar mass of the adsorbed GGM tend to be greater compared to those remaining in the continuous phase, ${ }^{18}$ greater surface load is achieved with fewer molecules. This knowledge leads us to hypothesize either that the size of GGM is of importance or that these larger molecules contain other functionalities, most likely PR, which aid their anchoring at the interface. Acetylation of hydroxyl groups alters the overall polarity of GGM, making them less polar. Acetyl groups may play a role in the functionality of GGM; however, they are not expected to carry the main responsibility for GGM's stabilization capacity. In addition, GGM contain only negligible portions of residual proteins; ${ }^{34}$ thus, their role in stabilization is considered insignificant.

PHWE GGM-stabilized emulsions had the greatest content of PR at the interface ( $2.6 \mu \mathrm{g} / \mathrm{kg}$ emulsion), especially of those that were ester bound to ethanol-soluble GGM, and at the same time had a unimodal droplet size distribution with smallest average droplet size and largest droplet surface area compared to emulsions prepared with the other types of GGM. In addition, PHWE emulsion remained extremely stable throughout the 6 weeks of the accelerated storage test. With the other types of GGM, a smaller 
amount of PR (0.4-1.7 $\mu \mathrm{g} / \mathrm{kg}$ emulsion), most of them being free or only weakly associated with GGM

356 (i.e., extractable with ethanol), were adsorbed at the droplet interface. These emulsions had larger average droplet sizes and were less stable. More importantly, when phenolic compounds were introduced as a separate extract (in eTMP+PE), emulsification was efficient, but the long-term physical stability of emulsion was not comparable to that of PHWE emulsion. Free PR may act as emulsifiers (i.e. facilitate emulsion formation), but may destabilize the interface during storage. ${ }^{35}$ Moreover, their partitioning between the continuous and interfacial phases is highly $\mathrm{pH}$ dependent due to the dissociation of the acid and hydroxyl groups. Most of the ester-bound PR, on the other hand, lack the acid group and thus remain neutral at a wide $\mathrm{pH}$ range.

PR may be bound to the monosaccharide units of GGM either via ester or ether bonds or via glycosidic bonds. $^{23,36}$ Part of these bonds may occur naturally, but they are also formed during the isolation processes. Each monosaccharide unit has several hydroxyl groups available for ester bonding, but GGM have only one site for glycosidic bonding, that is, at the reducing end of the molecule. Non-polar units attached to the polysaccharide backbone are considered to increase the amphiphilic nature of GGM and enable their anchoring to the oil droplet interface. Larger GGM chains have more possible sites available for ester linking and therefore a greater number of PR could be bound. Further increase in the amphiphilic nature would make these larger GGM molecules more susceptible to adsorb at the interface. Ester-bound PR would also enable even packaging of GGM parallel to the interface (Fig. 9) and therefore could lead to greater physical stability and protection against lipid oxidation, as shown for PHWE emulsions in this study. Glycosidically bound PR, on the other hand, may be located only at the reducing end of the GGM molecules and could therefore lead to perpendicular anchoring of GGM to the surface. Our hypotheses of orientation of GGM-PR assemblies on droplet interface are presented in Fig. 9. This orientation is hypothesized to form a thick, but porous interfacial layer due to steric hindrance caused by the GGM chains with attached galactopyranosyl side groups. It is likely that this perpendicular orientation and steric hindrance would lead to larger droplet size than if GGM chains were aligned with the interface, as 
380 demonstrated with sTMP and eTMP emulsions containing a considerable fraction of glycosidically

381 bound PR at the interface. In addition, a thick and porous layer could be responsible for the observed

382 flocculation of these emulsions. Flocculation may eventually lead to coalescence and increased average

383 droplet size. However, thick interfacial layer, if not too porous, could inhibit lipid oxidation by acting as

384 a steric barrier between the oxidation initiators and dispersed lipids, ${ }^{13-14}$ which could explain the

385 oxidative stability of sTMP emulsion. The presently hypothesized nature of GGM-PR assemblies at

386 droplet interface explains the anchoring of intermediate-sized GGM tails on the droplet surface by

387 lipophilic PR structures. This is analogic to protein anchors in gum Arabic ${ }^{4,5}$, with the difference that the

388 present GGM-PR assemblies were more efficient stabilizers than gum Arabic studied earlier ${ }^{18}$. Such

389 structural assemblies are suggested as guidelines in targeted isolation of bio-based hydrocolloid

390 structures with high functionality or in derivatization or synthesis of structural elements designed for

391 efficient interfacial stabilization.

392

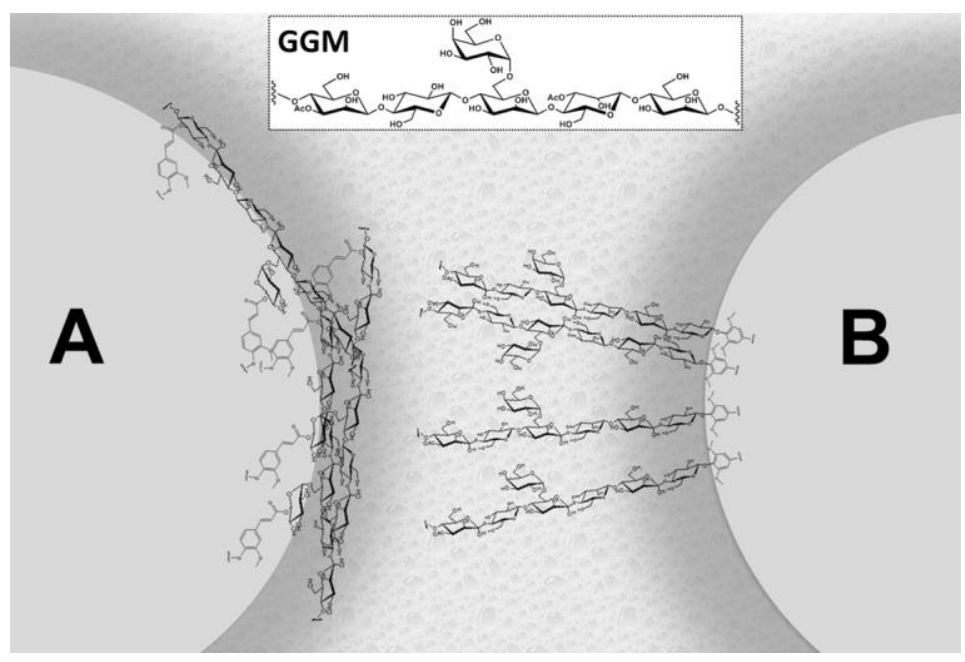

393 Figure 9. Simplified illustration of the hypothetical orientation of GGM containing ester-bound (A) and

394 glycosidically bound (B) phenolic residues at the interface of oil-in-water emulsion.

395 GGM may also stabilize emulsions via Pickering-type stabilization, that is, forming an interfacial layer

396 by assemblies. ${ }^{19}$ Assemblies comprise parallel-orientated non-branched carbohydrate chains, which are

397 associated via hydrogen bonding of the hydroxyl groups. ${ }^{37}$ Alternatively, PR may promote GGM-GGM 
associations due to their polarity difference with the aqueous medium or due to the hydrogen bonding of

399 PR. In Pickering-type stabilization, the size and orientation of the assemblies determine the droplet size 400 and thickness of the interfacial layer. ${ }^{10-11,19}$

401 The chemical structures of PR in the studied GGM samples varied from simple free aromatic phenolic 402 acids to polymers of flavonoids. Lignin is a polymer of ester, ether, glycosidically and carbon-bound 403 phenyl propanoid units. ${ }^{38}$ Lignin may exist in free form or it may be bound or associated to surrounding 404 hemicelluloses and cellulose forming LCC. ${ }^{23,36}$ In these complexes, lignin is connected to sugar units 405 mainly with hydroxycinnamyl or hydroxybenzyl units via glycosidic, ester or ether bonds. In base and acid hydrolyses of GGM, as used in the present work, various bond types existing between the carbohydrate chain and phenolic residues and between different phenyl units in the PR are cleaved. Thus, this technique provided information about the various groups of phenyl units present in GGM, even though it did not reveal their complex native structures. As lignin fragments in LCC seem to be mainly monomers and dimers, ${ }^{39}$ we hypothesize that the PR in the studied GGM are rather small units. The bond 411 type and composition of released PR clearly correlated with the stabilization capacity of GGM. 412 Hydroxycinnayls ester bound to ethanol-soluble GGM were most efficiently adsorbed to the oil droplet 413 interface. Emulsions containing higher amounts of hydroxycinnamyls appeared to be most stable.

414 Previous studies have proposed that PR are more likely to interact with hemicelluloses with higher 415 numbers of galactose side groups. ${ }^{23}$ If the residues are bound to galactose units, this could enable PR to 416 get in contact with the lipid surface despite the possible steric hindrance caused by rigid GGM. While 417 attaching to the non-polar medium, the PR would simultaneously anchor GGM to the interface.

418 The large interfacial area in emulsions predisposes lipids into enhanced contact with oxidation 419 initiators. In addition, flocculation may bring dispersed lipid droplets into close contact, enhancing the 420 transfer of lipid radicals and hydroperoxides from one droplet to another. This interaction may lead to 421 an increased oxidation rate. If the interfacial layer is sufficiently thick and/or dense, it acts as physical 422 barrier between active initiators and lipid species, while at the same time preventing further oxidation. ${ }^{13-}$ 
${ }^{14}$ In addition to acting as physical barrier, GGM containing PR may protect lipids against oxidation via

424 scavenging radicals and binding iron. ${ }^{40}$

425 Lipids were most stable against oxidation in PHWE emulsion that had the smallest average droplet 426 size, the largest surface area and the greatest content of PR, especially those that were bound to GGM, 427 among the studied emulsions. In contrast, lipid oxidation was most intensive in eTMP emulsion which 428 had larger average droplet size, smaller surface area and contained less PR compared to the other 429 emulsions. ${ }^{20}$ The addition of phenolic compounds as a separate extract (eTMP+PE) improved oxidative 430 stability, but not to the level of PHWE emulsion. Thus, PR, especially those associated with GGM, 431 seemed to have a significant role in the stabilization of lipids against oxidation. Lipid oxidation and 432 physical stability are also expected to be interlinked, for example, oxidized lipid droplets may be more 433 prone to Ostwald ripening due to their increased miscibility with the continuous phase after increased 434 polarity.

435 In all of the studied emulsions, decrease in the contents of PR occurred during the storage. The changes 436 were most evident in the ethanol-soluble fractions, both in free PR and in ester-bound PR. In PHWE and 437 sTMP emulsions, changes in the PR occurred mainly in the continuous phase. At the same time, these 438 emulsions were most stable against oxidation. In eTMP+PE emulsion, containing only minor amount of 439 GGM-bound PR, decrease in phenolic compounds was most apparent at the interface. In addition, lipid 440 oxidation was greater in this emulsion compared to PHWE and sTMP emulsions. Adsorbed free phenolic 441 compounds may have deattached from the interface during storage, leading to a decrease in their contents 442 and possible destabilization of the interface. Reactions of PR with radicals may also promote 443 polymerization of GGM and formation of interaction products with lipids. Even if the polymerized or 444 interacted GGM would remain at the droplet interface, the number of antioxidant active PR would be 445 reduced. Decrease in the coverage of the interface and reduced content of antioxidants at the site of 446 radical formation could have led to increased lipid oxidation. 
447 In addition to the physical stabilization of emulsions and its protective effect against lipid oxidation,

448 GGM-bound hydroxycinnamyls located at the interface could act as antioxidants. Phenolic compounds 449 may scavenge primary carbon-centered lipid radicals and stop the chain reaction before it escalates and 450 leads to elevated oxidation. ${ }^{41} \mathrm{PR}$ in the continuous phase, on the other hand, could have functioned via 451 binding or reducing transition metals and thus inhibiting oxidation. Polysaccharides themselves may also 452 retard lipid oxidation in emulsions. ${ }^{2,6}$ The action may be due to increment of viscosity or due to transition 453 metal binding. Polysaccharides have also been proposed to scavenge carbon and hydroxyl radicals. ${ }^{42-43}$ 454 Other wood extractives, such as triterpenes and sterols, may also possess antioxidant activity. 455 However, their contents in wood are relatively low, usually less than $1-2 \%$ in heartwood and sapwood..$^{30}$ 456 Half of this content (i.e., $0.5-1 \%$ ) may have been present in the studied GGM fractions. ${ }^{25}$ If this was the 457 case, their content could have been significant in eTMP but negligible in PHWE. Yet, PHWE emulsions 458 were more stable against oxidation than eTMP emulsions. Knotwood extract of Norway spruce has been 459 reported to contain considerable amounts of lignans, 7-hydroxymatairesinol being the predominant one $460(70-85 \%){ }^{44}$ These compounds may have been responsible for the increased oxidative stability of 461 eTMP+PE emulsion.

462 Current results support the use of natural non-purified GGM fractions in emulsion stabilization. 463 Extraction of a sufficient amount of GGM containing bound PR will give GGM superior functionality. 464 Regarding applications, it is noteworthy that the color of GGM extracts is correlated to the content and 465 composition of lignin residues. Obtained results provide crucial information for industry, which is 466 currently developing non-hazardous and economical processes for the isolation of GGM. For example, 467 with flow-through pressurized hot water extraction, the size of GGM and content of PR can be 468 controlled. ${ }^{25,45}$ Moreover, the composition of PR and thus also the color of the extract may be adjusted 469 by extraction temperature and time.

\section{Conclusions}


471 We showed that the phenolic profile in GGM contribute to their interfacial functionality. PR act as 472 vehicles that enable the anchoring of GGM into the oil droplet interface. GGM-PR assemblies provide 473 steric stabilization against physical breakdown and antioxidants against lipid oxidation. The composition 474 of PR in natural GGM extracts can be tailored by sustainable and economical isolation processes. GGM 475 are multifunctional plant-based emulsifiers and stabilizers exhibiting both physical and chemical 476 stabilization capacity over extended storage time. According to the hypothesis, we demonstrated that by 477 retaining interactions between isolated GGM and PR, stabilization is achieved without adding other 478 surfactants or antioxidants. Previously known emulsifiers are typically small-molecular amphiphilic 479 surfactants, such as Tween $20,{ }^{46}$ whereas macromolecular hydrocolloids are mainly used as viscosity480 modifying stabilizers. ${ }^{6-9}$ The present work suggests that lipophilic structures bound with intermediate481 sized polysaccharide tails result in highly efficient interfacial stability. Superior physical stabilization 482 and protection against lipid oxidation compared to e.g. gum Arabic ${ }^{18,20}$ or Tween $20{ }^{46}$ was obtained by 483 one-ingredient formulation and one-step emulsification process. The shelf life of PHWE GGM484 stabilized emulsions, in terms of physical and oxidative stability, at room temperature would be up to 8 485 months. Correspondingly, the shelf life would be prolonged up to 1.5 years at cooled conditions, such as 486 in a refrigerator. Even with less efficient GGM fractions, the emulsions would remain stable for 1-4 487 months. This high and long-term emulsion stability and abundant availability of raw materials makes 488 "spruce gum" an attractive future alternative for conventionally used emulsifiers and stabilizers in 489 various technical, pharmaceutical and food applications. Future work includes investigation of 490 continuous phase composition and dispersed phase volume fraction on the stabilization capacity of 491 GGM. Furthermore, understanding the functionality of LCC at the emulsion interface provides 492 perspectives for future tailoring of both natural and synthetic compounds for efficient stabilization of 493 dispersed systems.

\section{Conflicts of interest}


495 There are no conflicts to declare.

\section{Funding Sources}

497 The research was funded by the Academy of Finland (project numbers 268144 and 305517) and by the 498 Jane and Aatos Erkko Foundation.

\section{ACKNOWLEDGMENTS}

500 The Academy of Finland (project numbers 268144 and 305517) and Jane and Aatos Erkko Foundation

501 are gratefully acknowledged for funding the study. We thank Docent Anna-Maija Lampi for fruitful

502 discussions and Suvi Teräslahti for the optical microscopic image of eTMP GGM-stabilized emulsion.

\section{REFERENCES}

504 1. P. C. Hiemenz and R. Rajagopalan, Principles of Colloid and Surface Chemistry, 3rd ed., Marcel

505 Dekker, Inc., New York, 1997.

506 2. D. J. McClements and E. A. Decker, J. Food Sci., 2000, 65, 1270.

507 3. R. Charoen, A. Jangchud, K. Jangchud, T. Harnsilawat, O. Naivikul and D. J. McClements, J. $508 \quad$ Food Sci., 2011, 76, E165.

509 4. E. Dickinson, Food Hydrocolloids, 2003, 17, 25.

510 5. E. Dickinson, Food Hydrocolloids, 2009, 23, 1473.

511 6. D. Paraskevopoulou, D. Boskou and A. Paraskevopoulou, Food Chem. 2007, 101, 1197.

512 7. S. Kokubun, M. P. Yadav, R. A. Moreau and P. A. Williams, Food Hydrocolloids, 2014, 41, 164.

513 8. U. S. Schmidt, K. Schmidt, T. Kurz, H.-U. Endreß and H. P. Schuchmann, Food Hydrocolloids, $514 \quad 2015, \mathbf{4 6}, 59$.

515 9. L. Bai, S. Huan, Z. Li and D. J. McClements, Food Hydrocolloids, 2017, 66, 144. 
516 10. C. C. Berton-Carabin and K. Schroën, Annu. Rev. Food Sci. Technol., 2015, 6, 263.

517 11. Z. Hu, H. S. Marway, H. Kasem, R. Pelton and E. D. Cranston, ACS Macro Lett., 2016, 5, 185.

518 12. C. C. Berton-Carabin, A. Schröder, A. Rovalino-Cordova and K. Schroën, Eur. J. Lipid Sci.

$519 \quad$ Technol., 2016, 118, 1630.

520 13. T. Waraho, D. J. McClements and E. A. Decker, Trends Food Sci. Technol., 2011, $22,3$.

521 14. C. C. Berton-Carabin, M.-H. Ropers, C. Genot, Compr. Rev. Food Sci. F., 2014, $13,945$.

522 15. D. Guzey and D. J. McClements, Adv. Colloid Interface Sci., 2006, 128-130, 227.

523 16. D. O. Grigoriev and R. Miller, Curr. Opinion Colloid Interface Sci., 2009, 14, 48.

524 17. M. V. Lomova, G. B. Sukhorukov and M. N. Antipina, ACS Appl. Mater. Interfaces, 2010, 2, $525 \quad 3669$.

526 18. K. S. Mikkonen, C. Xu, C. Berton-Carabin and K. Schroën, Food Hydrocolloids 2016a, 52, 615.

527 19. K. S. Mikkonen, D. Merger, P. O. Kilpeläinen, L. Murtomäki, U. S. Schmidt and M. Wilhelm, Soft $528 \quad$ Matter, 2016b, 12, 8690.

529 20. M. Lehtonen, S. Teräslahti, C. Xu, M. P. Yadav, A.-M. Lampi and K. S. Mikkonen, Food $530 \quad$ Hydrocolloids, 2016, 58, 255.

531 21. L. Pitkänen, M. Heinonen and K.S. Mikkonen, Submitted.

532 22. S. Willför, K. Sundberg, M. Tenkanen and B. Holmbom, Carbohydr. Polym., 2008, 72, 197.

533 23. M. Lawoko, PhD thesis, KTH Royal Institute of Technology, 2005.

534 24. P. Laine, A.-M. Lampi, M. Peura, J. Kansikas, K. S. Mikkonen, S. Willför, M. Tenkanen and K. $535 \quad$ Jouppila, J Agric. Food Chem., 2010, 58, 981.

536 25. P. O. Kilpeläinen, S. S. Hautala, O. O. Byman, L. J. Tanner, R. I. Korpinen, M. K.-J. Lillandt, A. 537 V. Pranovich, V. H. Kitunen, S. M. Willför and H. S. Ilvesniemi, Green Chem., 2014, 16, 3186.

538 26. M. Plaza and C. Turner, Trends Anal. Chem., 2015, 71, 39.

539 27. I. Sumerskiy, A. Pranovich, B. Holmbom and S. Willför, J. Wood Chem. Technol., 2015, 35, 387.

540 28. S. Willför, P. Rehn, A. Sundberg, K. Sundberg and B. Holmbom, Tappi J., 2003a, 2 , 27. 
29. C. Xu, S. Willför, K. Sundberg, C. Petterson and B. Holmbom, Cell Chem. Technol., 2007, 41, 51.

542 30. S. Willför, J. Hemming, M. Reunanen, C. Eckerman and B. Holmbom, Holzforschung, 2003b, 57, 27.

544 31. A.-M. Lampi, L. Kataja, A. Kamal-Eldin and V. Piironen, J. Am. Oil Chem. Soc., 1999, 76, 749.

545 32. Folin-Ciocalteu Index. OJEC, 1992, p. 178.

546 33. P. Kylli, L. Nohynek, R. Puupponen-Pimiä, B. Westerlund-Wikström, T. Leppänen, J. Welling, E.

547 Moilanen and M. Heinonen, J. Agric. Food Chem., 2011, 59, 3373.

548 34. K. S. Mikkonen, M. Tenkanen, P. Cooke, C. Xu, H. Rita, S. Willför, B. Holmbom, K. B. Hicks

549 and M. P. Yadav, Food Sci. Technol., 2009, 42, 849.

550 35. K. Schwarz, E. N. Frankel and J. B. German, Fett/Lipid, 1996, 98, 115.

551 36. N. Giummarella and M. Lawoko, Sustainable Chem. Eng., 2017, 5, 5156.

552 37. L. Pitkänen, $\mathrm{PhD}$ thesis, University of Helsinki, 2011.

553 38. G. Henriksson, in Pulp and Paper Chemistry and Technology, Volume 1, Wood chemistry and

554 wood biotechnology, ed. G. Henriksson, G. Gellerstedt and M. Ek, De Gruyter, Berlin, 2009,

$555 \quad$ Chapter 6, p. 121-146.

556 39. X. Du, G. Gellerstedt and J. Li, Plant J., 2013, 74, 328.

557 40. A. Ebringerová, Z. Hromádková, V. Hř́ibalová, C. Xu, B. Holmbom, A. Sundberg and S. Willför, 558 Int. J. Biol. Macromol., 2008, 42, 1.

559 41. E. Choe and D. B. Min, Compr. Rev. Food Sci. F., 2009, 8, 345.

560 42. S. J. Blanksby and G. B. Ellison, Acc. Chem. Res. 2003, 36, 255.

561 43. E. Machová, A. Č́ížová and P. Bystrický, Cabohydr. Polym., 2014, 112, 603.

562 44. B. Holmbom, C. Eckerman, P. Eklund, J. Hemming, L. Nisula, M. Reunanen, R. Sjöholm, A.

563 Sundberg, K. Sundberg and S. Willför, Phytochem. Rev., 2003, 2, 331. 
564 45. K. Leppänen, P. Spetz, A. Pranovich, K. Hartonen, V. Kitunen and H. Ilvesniemi, Wood Sci. 565 Technol., 2011, 45, 223.

566 46. M. Heinonen, K. Haila, A.-M. Lampi and V. Piironen. J. Am. Oil Chem. Soc., 1997, 74, 104. 